\title{
Superando los reduccionismos en la gestión de sistemas de información y documentación
}

\author{
Francisco Javier García Marco \\ Universidad de Zaragoza
}

\subsection{Resumen}

Se analizan algunos de los retos actuales en la gestión de sistemas de información a partir de la síntesis y discusión de los estudios publicados en la revista Scire (vol. $9, \mathrm{n}^{\circ} .1$ ). La tecnología aporta herramientas potentísimas y las técnicas intelectuales un marco de trabajo seguro, pero, al final, gestionar sistemas de información y documentación es un problema centrado en la "gente" —en sus necesidades e intereses, en sus capacidades e interrelaciones - ; en definitiva, en animar, servir y racionalizar sistemas humanos en lo que concierne a la necesidad compartida de preservar y comunicar la memoria común. Es precisamente el factor humano el que otorga el sentido de misión a la biblioteca, que no se limita a proporcionar servicios, sino que además configura un espacio de convivencia en el que las comunidades humanas pueden concentrarse en los aspectos relacionados con la transmisión del conocimiento y celebrar la memoria colectiva. Los reduccionismos - gerencial, tecnológico, social, ético...- pueden servir para guiar la especialización y focalizar prioridades, pero a la larga solo una visión sistémica y humanística va a permitir mantener la nave de la práctica profesional, la enseñanza y la investigación de nuestro área en la estela de las disciplinas clásicas. En una época de cambio, la gestión estratégica de recursos humanos, especialmente en lo que se refiere a los aspectos formativos, es una de las líneas prioritarias para realizar este enfoque.

Palabras clave: Sistemas de información y documentación. Bibliotecas. Gestión. Gestión de la calidad. Evaluación. Recursos humanos. Formación. Misión. Filosofía. Evolución profesional. Adaptación al cambio.

\subsection{Abstract}

Some of the current challenges in the management of information systems are analyzed, building on the synthesis and discussion of the studies published in the Scire. $9: 1$ (en.-jun. 2003) 9-19. 
first number of the ninth volume of the journal Scire. Technology contributes with powerful tools and intellectual techniques ensure a safe frame of work; but, eventually, managing information and documentation systems is a problem centred in people, in their needs and interests, and in their capabilities and interrelations. Building information and documentation systems is about animating, serving and rationalizing human systems in those specific aspects concerned with the shared need of preserving and communicating their common memory. It is the human factor that gives libraries and information centres their sense of mission, because they not only give services, but also configure spaces of conviviality in which human communities concentrate in the aspects related to the transmission of the knowledge and celebrate their collective memory. Reductionisms - managerial, technological, social, ethical, etc. - are useful for guiding specialization and focusing priorities, but in the long trend only a systemic and humanistic perspective will keep the professional, educational and research practices in the trail of the classical disciplines. In a age of change, strategic management of human resources, especially in those aspects connected with education and training, must be carefully considered in order to accomplish such a mission statement.

Keywords: Information and documentation systems. Libraries. Management. Quality management. Evaluation. Human resources. Education and training. Mission. Philosophy. Professional evolution. Adaptation to change.

\section{Introducción}

En los últimos años se está está produciendo una corrección en la tendencia central del discurso académico y profesional. El énfasis se está trasladando desde la tecnología a la preocupación por la gestión integral con un acento en la interacción social y el factor humano. Si los setenta fueron la década del análisis, los ochenta la década de la automatización y los noventa la década de Internet, es posible que nos acerquemos $-i$ o estamos entrando ya? - en una nueva etapa en la cual la principal preocupación sea la gestión de una profesión cuyos conocimientos, entornos y áreas de actuación son cada vez más complejos, más diversificados y también - en correspondencia - más interconectados.

El conjunto de estudios que componen este número tiene precisamente como nexo principal de unión su preocupación por llamar la atención desde distintas especialidades y ámbitos de actuación por el problema de la gestión de entornos complejos - redes de cooperación y grandes organizaciones - afectados por el impacto de los cambios tecnológicos y la globalización. En estos entornos complejos el factor que merece especial atención son los actores humanos, y, en relación con ese énfasis, la necesidad de una permanente evaluación y renovación de los programas formativos. Estos estudios ponen a la "gente" en el centro de la actividad y la investigación informativo-documental. De alguna mera, son

Scire. $9: 1$ (en.-jun. 2003) 9-19. 
representativos del regreso de la "gente" al centro del discurso informativo-documental que, por un tiempo, había sido ocupado por el inevitable protagonismo de los revolucionarios sistemas informáticos.

Y es que, en definitiva, el funcionamiento de los sistemas de información no depende solo de técnicas y herramientas, sino también, y con igual o mayor importancia, de articulación de los intereses de las personas -individualmente o en grupo- cuyo interés efectivo y compromiso hacen su existencia posible y funcional - si se quiere valiosa - . La revolución de las tecnologías de la información ha modificado el panorama social y laboral, y tras la urgencia tecnológica llega la hora de evaluar su impacto, reparar daños, aprovechar oportunidades y centrar la situación desde un punto de vista sistémico.

El panorama de los sistemas de información y documentación ha cambiado notablemente con la revolución informática agudizando tendencias que venían desarrollándose, no obstante, a ritmo lento, y de las que interesa ahora resaltar tres. En primer lugar, la informatización ha esponjado y aligerado de manera increíble las labores administrativas cuestionando de raíz la filosofía de su organización laboral. En segundo lugar, la red ha hecho inevitable a la par que posible la cooperación y la integración de sistemas en una escala apenas pensable hace dos décadas, a la vez que ha convertido los servicios de documentación en una de las actividades más globalizadas del planeta. Por fin, en tercer lugar, la información ha empezado a fluir de una manera asombrosa por todo el planeta a través de los nuevos medios de comunicación, dando una nueva dimensión a las tareas documentales de organizar, conservar y difundir los registros del conocimiento. En el trabajo que cierra este volumen planteamos algunos los retos que, a nuestro juicio, caracterizan el momento actual: la integración de conocimientos en un entorno globalizado, la dinámica especialización-interdisciplinariedad, el filtrado y modulado de la información, el conocimiento de calidad, el aprendizaje continuo y la gestión de las redes de conocimiento (García, 2003). La tecnología aporta herramientas potentísimas y las técnicas intelectuales un marco de trabajo seguro, pero, al final, gestionar sistemas de información y documentación es un problema centrado en la "gente" - en sus necesidades e intereses, en sus capacidades, en sus interrelaciones - , centrado en definitiva en animar, servir y racionalizar sistemas humanos en lo que concierne a la necesidad compartida de preservar y comunicar la memoria común.

Los estudios que se presentan a continuación inciden precisamente desde disintas ópticas y ámbitos temáticos en la importancia del factor humano dentro de los sistemas de información y documentación. 


\section{La gestión sistémica del impacto de las tecnologías en los sistemas de información y documentación}

El trabajo de José Vicente Rodríguez Muñoz abre el volumen se enfrenta con el problema de la gestión de la complejidad en el campo de la absorción de la innovación tecnológica en las organizaciones. En su artículo muestra la naturaleza inherentemente inestable de las organizaciones humanas, a las que el cambio tecnológico añade un plus de complejidad que en muchos casos amenaza el difícil equilibrio del sistema. Esta realidad lleva a Rodríguez Muñoz (2003, p. 35) a llamar la atención sobre una articulación distinta del problema de las tecnologías en su aplicación informativo-documental a la que es usual en el discurso de nuestro área, concentrado en las aplicaciones funcionales:

La verdadera complejidad de las tecnologías de la información, en consecuencia, no reside tanto en la articulación funcional - su usabilidad, su diversidad o su sofisticación-, sino en las profundas transformaciones que desencadena en los modos de organización de los sistemas humanos.

Rodríguez Muñoz articula con ayuda de sus fuentes en un discurso teórico convincente y riguroso la desazón de informáticos, bibliotecarios, documentalistas y, en general, de todos los profesionales de la información, con el ritmo al que evoluciona la tecnología. Si a principios de los años ochenta del siglo pasado resultaba posible dominar o al menos conocer con cierta profundidad los principales sistemas operativos, los lenguajes de programación y las aplicaciones informáticas, la dinámica actual de obsolescencia, especialización y sofisticación creciente en los sistemas informáticos ha hecho de su uso una actividad compleja y estresante, tanto más cuanto más implicado se esté en tareas relacionadas con su administración, planificación, diseño, programación o adquisición. Pero los problemas personales no son más que el síntoma de un mal sistémico: la lucha de las organizaciones sociales por asimilar el cambio tecnológico y sus consecuencias.

Es por ello que la necesidad que plantea Rodríguez Muñoz de asumir la gestión de tecnología desde el liderazgo de la organización y de forma planificada y consciente ha de constituir una de las citas ineludibles de la agenda gerencial e investigadora de los próximos años. Dicho en sus propias palabras, el cambio tecnológico y los retos que ésta plantea en la organización y sus individuos, "demanda cada vez más una complejización de los sistemas sociales capaz de absorber el excedente de incertidumbre que las tecnologías aportan" (ibidem, p. 36).

\section{Gestionando las arquitecturas de información corporativas y sus taxonomías como un problema sistémico}

Desde un punto de vista distinto - el de la organización del conocimiento-, Alan Gilchrist incide también en el problema del carácter sistémico de la gestión

Scire. $9: 1$ (en.-jun. 2003) 9-19. 
de la información, en este caso focalizado en sus aspectos globales - diseño, planificación y sostenibilidad-, esto es, en la "arquitectura de información". Gilchrist (2004) aborda un nuevo sistema de organización del conocimiento, las taxanomías, que a diferencia de los tesauros y las clasificaciones - aunque inspirándose en ambas - se centran en la información interna de la organización y pretenden integrar el resto de sus sistemas terminológicos en un sistema coherente capaz de organizar el acceso y la recuperación en los nuevos sistemas de información complejos e integrados que forman el sistema neurológico de las organizaciones y cuyo aspecto más visible son los portales corporativos. Las taxonomías corporativas son, en realidad, lenguajes documentales multimedia que vuelven a integrar el acceso alfabético - en listas o mediante palabras clave- y sistemático, así como las necesidades y perspectivas de búsqueda de usuarios y administradores del sistema, compatibilizándolas con la potencia técnica que ofrecen las nuevas herramientas informáticas.

Pero más allá del tema de su artículo, interesa como Gilchrist lo aborda. Y lo hace basándose en una herramienta eminentemente sistémica, el llamado diamante de Leavitt (1965), un modelo inventado a mitad de los sesenta para dar cuenta de las relaciones entre los sistemas técnicos y socio-técnicos en el seno de las organizaciones y que contempla la relación entre la estructura - entendida como los elementos más estables de la organización, por ejemplo, los objetivos y las normas -, los procesos, las herramientas y la gente. No sorprende el reconocimiento que hace Gilchrist de que la "gente" es "quizá, el área más difícil de considerar, ya que abarca el orden completo de los problemas humanos, incluyendo las características y aspiraciones individuales, y el modo en que los individuos se relacionan con los grupos y las organizaciones en su conjunto". Al final, el ideal de la taxonomía como un sistema de organización y recuperación del conocimiento integral (ibidem, p. 46):

[...] requiere la cooperación generalizada y la habilidad del equipo de arquitectos de la información para superar todos aquellos problemas políticos a los que las organizaciones son tan proclives. El equipo debe establecer los principios de gobierno y propiedad; todos los implicados deben tener la posibilidad de participar y defender sus intereses y perspectivas; y se requiere el liderazgo de un "campeón" del proyecto del más alto nivel.

También en el trabajo de Miguel Ángel López-Alonso (2003) - que aborda los aspectos terminológicos, normativos e informáticos del desarrollo de una herramienta terminológica común para la red española de centros autonómicos de información juvenil - aflora el problema organizativo. Una de las lecciones de la experiencia de construcción de un tesauro de información juvenil es la "ardua tarea" que conlleva "la homologación de las colaboraciones terminológicas de los responsables de los diecisiete centros autonómicos españoles de información

Scire. 9 : 1 (en.-jun. 2003) 9-19. 
juvenil, debido mayoritariamente a la falta de integración previa de sus sistemas de información." (ibidem, p. 52). El trabajo de López Alonso es una muestra de la necesidad de nuestras unidades de desarrollar su trabajo de forma conjunta en beneficio del usuario, creando portales y herramientas terminológicas de acceso comunes. Por lo demás, el enfoque al trabajo cooperativo sobre la red Internet desarrollado en el marco de estándares abiertos es una apuesta adecuada y necesaria en línea con las últimas tendencias. Graciela Barcalá de Moyano (2003) en su informe del proyecto "Memoria Abierta" también señala en sus conclusiones que el mayor factor de complejidad que tuvo que afrontar fue la gestión del factor humano interno y externo. Dicho en sus palabras: "uno de los mayores desafíos fue la elección de los medios organizacionales, técnicos y humanos, la integración de las tareas del equipo de profesionales - bibliotecarios, documentalistas e informáticos - y la búsqueda de las condiciones para servir de vehículo entre las autoridades de cada organismo y los técnicos" (2003, p. 149). Su artículo relata un interesantísimo estudio de caso que muestra como los profesionales de la información y la documentación pueden abordar con éxito proyectos en nichos de trabajo completamente nuevos - en este caso, las organizaciones no gubernamentales de derechos humanos de la Argentina - superando los problemas de viabilidad económica con imaginación y el recurso a fuentes de financiación no habituales, sin perjuicio de la calidad y profesionalidad del proyecto.

\section{Los recursos humanos como aspecto clave de la planificación estratégica}

El artículo de Juan José Fuentes Romero (2003) es decisivo en la línea que estamos analizando. Al abordar la planificación estratégica de las bibliotecas nacionales - cuyos problemas son muchas veces los de todas las bibliotecas, pero a una escala proporcional a su tamaño y complejidad-, Fuentes afirma sintéticamente que "de todos los factores que intervienen en la buena marcha de una biblioteca probablemente sea la adecuada planificación de sus recursos humanos uno de los más importantes, si no el que más, en el mantenimiento de unas condiciones óptimas de trabajo y en el logro de los objetivos que lógicamente se desea alcanzar" (ibidem, p. 58). Sin embargo, lejos de hacer gravitar el problema humano sobre sí mismo, liga su abordaje al de planificación económica. Es difícil gestionar recursos humanos cuando la tarea aumenta - crecimiento exponencial de la documentación, recursos electrónicos, mayor necesidad de formación del personal y los usuarios, etc. - , el personal disminuye, los presupuestos permanecen estables y los gastos en sistemas informáticos crecen en ausencia de nuevos recursos económicos. Es cierto que las tecnologías han multiplicado la productividad de las tareas relacionadas con la gestión de la información, pero es necesario evaluar hasta qué punto. En particular, hay que determinar si no se están tensionando

Scire. 9 : 1 (en.-jun. 2003) 9-19. 
irracionalmente nuestros sistemas de información al privarles de una financiación adecuada, sin la cual es imposible la eficacia por mucho que se ajuste la eficiencia. Fuentes trae a colación al respecto las clarificadoras Pautas del Consejo de Europa sobre legislación y política bibliotecaria (Guidelines..., 1998).

Pero, de forma recíproca, hay que evitar caer en el discurso contrario. Los recursos solo dan fruto en relación directamente proporcional a la capacidad de utilizarlos adecuadamente y a la oportunidad de su uso. La revolución tecnológica ha cambiado el concepto de la eficacia y la eficiencia en el ámbito de las unidades de información y documentación. Las oportunidades y retos planteados por las nuevas tecnologías en las bibliotecas han provocado una revolución en los roles tradicionales del personal bibliotecario (García Marco, 1998), que solo puede resolverse a través de la formación profesional. Una de las técnicas que se ha mostrado más eficaz en términos generales en el campo de la formación profesional ha sido el enfoque orientado al "desarrollo de competencias". A partir de la experiencia de la Biblioteca Nacional de Canadá, Fuentes revisa la utilidad de el conjunto de herramientas utilizado, especialmente del perfil de competencias, el plan de entrenamiento y el inventario de habilidades.

\section{Gestión de la calidad}

Waldomiro Vergueiro y Telma de Carvalho (2003) abordan para la realidad brasileña un tema que sigue siendo protagonista de la práctica y la literatura sobre sistemas de información y documentación: la gestión de la calidad. Muestran como en Brasil la puerta estrecha - y cara - que forman las ISO 9000 apenas ha sido franqueada por alguna biblioteca, y que, sin embargo, el generalizado interés por la calidad de los servicios bibliotecarios - muchas bibliotecas, aunque se encuentran lejos de desarrollar un sistema de calidad integral, utilizan técnicas como Just-in-Time, Kaisen, Control de la Calidad Total, Mantenimiento Productivo Total y $5 \mathrm{~S}-$ se está disciplinando a través de iniciativas como los premios a la calidad basados en el modelo de siete criterios de la Malcolm Baldrige Nacional Quality Award, de Estados Unidos, que constituyen en opinión de nuestros autores - la cual compartimos - un entrenamiento válido para procesos más complejos y laboriosos de certificación internacional.

La situación en España no está tan alejada de la brasileña. En los últimos años algunas bibliotecas han conseguido la certificación ISO 9000, como el Servicio de Bibliotecas de la Universidad Autónoma de Barcelona o la Biblioteca Pública de Tarragona. Por otra parte, el gobierno central y los de algunas comunidades autónomas - Cataluña, Andalucía... - han promovido procesos de evaluación - basados en un primer ciclo interno y un segundo externo - con el objetivo de promocionar la cultura de la calidad. La metodología se suele basar en el modelo de excelencia de la European Foundation for Quality Management (EFQM), muy

Scire. $9: 1$ (en.-jun. 2003) 9-19. 
próximo a los siete criterios de la Malcolm Baldrige Nacional Quality Award, y culmina en un reconocimiento o una certificación.

En la actualidad este modelo está dando en general resultados muy positivos, aunque se plantean críticas relacionadas con la necesidad de definir claramente que se pretende conseguir con cada herramienta de promoción de la calidad, especialmente a medio y largo plazo. Y es que, al final, cualquier proceso de calidad, por muy orientado a la promoción que esté, culmina en un juicio que divide a aquéllos que cumplen los criterios de los que no los cumplen. Para que los resultados no sean traumáticos $-\mathrm{y}$, en definitiva, contraproducentes desde una lógica social elevada - es necesario que se cumplan como mínimo cuatro condiciones: a) que los criterios y - lo que en la práctica suele ser más importante - las metodologías de contrastación estén suficientemente claros y consensuados; b) que las bibliotecas puedan contar con asesoría y asistencia durante todo el proceso; c) que la evaluación externa sea respetable, exigente y bien documentada; y d) que las convocatorias estén permanentemente abiertas. El apoyo es crítico, pues en ocasiones las bibliotecas fracasan a pesar de ofrecer un servicio de calidad por carecer del personal formado - o muchas veces simplemente de suficiente personal - para liderar, coordinar y documentar todo el proceso. En este sentido, es posible también que fuera mejor dividir el papel de asesores y jueces que tienen muchas veces los comités externos, asignado esas funciones a dos comités distintos formados por personas diferentes. Por otra parte, aunque la competitividad y el prestigio son una motivación importante que los premios y las certificaciones ofrecen a los centros más ambiciosos; desde el punto de vista de la salud global del sistema en su conjunto, la promoción de los mejores no debe ser una alternativa frente al desarrollo de una cultura de la calidad generalizada y compartida; sino que ambos abordajes pueden y deben ser complementarios.

A pesar de sus fallos, los procesos en marcha han conseguido iniciar un camino, elevar el problema del desarrollo sistemático de la calidad a un punto más alto dentro de la agenda de nuestras organizaciones y, sin duda, contienen las bases para su perfeccionamiento posterior. Es necesario por parte de los responsables mejorar el sistema y dar cuenta de las críticas y sugerencias; y por parte de los evaluados, reconocer que en el momento actual de desarrollo del proceso los juicios deben considerarse dentro de todas sus limitaciones.

Ahora bien, la cultura de la calidad no debe limitarse solo a la práctica profesional, sino implicar a la academia. Cintia Braga Ferreira, Maria Bernadete Malerbo y Márcia Regina Silva (2003) abordan el problema de calidad de las tesis de maestría y doctorado de forma experimental a través de uno de los parámetros de calidad utilizados para su evaluación: la calidad de sus referencias y citas. Los resultados son preocupantes - casi un cuarto de las referencias contienen errores graves - en la medida en que la calidad de las citas suele correlacionar con la

Scire. 9 : 1 (en.-jun. 2003) 9-19. 
calidad global del trabajo de investigación. Kátia Corina Vieira (2003) plantea una posible raíz de estos problemas al señalar que una educación primaria y secundaria - ¿universitaria en ocasiones? - permite que los alumnos pasen curso tras curso con trabajos en los que se limitan a copiar del profesor y de ciertas enciclopedias, libros - y cada vez más de Internet - sin elaborar ni reflexionar ni, mucho menos, reconociendo créditos.

\section{La formación profesional y el futuro de la profesión}

En un análisis objetivo - que no desapasionado - y serio, Miriam Vieira da Cunha (2003) aborda de nuevo los problemas del cambio en el perfil del profesional de la información y del acecho de la desprofesionalización. Pero no lo hace desde un centraje absoluto en nuestra profesión, sino que lo plantea desde una óptica comparada en relación con otras actividades laborales y desde la perspectiva teórica de la Sociología de las Profesiones de Abbott y MacGuire. Desde estos presupuestos teóricos, la evolución de cada profesión es vista en función de los servicios que presta a la sociedad, en cuya eficacia reside en último término la única promesa de éxito. Cunha muestra en relación con este principio como, cuando la demanda de sus clientes sobrepasa a las posibilidades de los miembros de una profesión, otras llenan el vacío. Las tecnologías de la información han revolucionado el mundo de los profesionales de la información, poniendo en cuestión sus prioridades, métodos, competencias y enfoques, popularizando habilidades informacionales antes exclusivas de las profesiones en cuestión; a la par que ofrece multitud de nuevas oportunidades al generalizar el uso de la información, puesto que todas las profesiones están sufriendo problemas en la gestión y uso de la información. El secreto está en la línea del estudio presentado por Fuentes (2003) en aprovechar las oportunidades desarrollando sus habilidades y conocimientos, y también en potenciar sus alianzas con otras profesiones. "En un ambiente en mutación permanente, la capacidad de aprender continuamente es esencial." (Cunha, 2003, p. 92).

Por su parte, Kátia Corina Vieira (2003) nos plantea en toda su crudeza el problema de que aprender a aprender no se puede improvisar, y que el planteamiento acelerado de los cursos de metodología de la investigación no bastan para suplir esta carencia en el nivel universitario. Apoyándose en el pensamiento de Saviani y Freire nos muestra también que el aprendizaje verdadero comprometido con los hechos y no tanto con la autoridad del profesor solo es posible desde la promoción del pensamiento crítico y la autonomía y dignidad personal. Muchos de los problemas que detecta en la enseñanza primaria y secundaria deberían ser objeto también de la reflexión de los profesionales de la formación universitaria.

Precisamente, Ely Francina Tannuri de Oliveira (2003) y Vânia Martins Bueno de Olivieira Funaro y Caludio Marcondes de Castro Filho (2003) nos

Scire. $9: 1$ (en.-jun. 2003) 9-19. 
ofrecen sendos diagnósticos de la enseñanza en nuestro área (Agencia Nacional de Evaluación Acreditación, 2004). Oliveira presenta un completo diagnóstico de la enseñanza de la información y la documentación en el MercoSur desde el punto de vista de la convergencia educacional, otro asunto que está ahora en el candelero también en España con el proceso de homologación europea de los planes de estudio universitarios, conocido como "proceso de Bolonia". El estudio de Oliveira reafirma, como el de Vieira en otro contexto, la necesidad de mantener unidas investigación y enseñanza en lo que se refiere tanto al perfil de los docentes como al currículum de los alumnos. También muestra el imparable desarrollo de la formación continua "como base para que el profesional pueda actualizarse y amoldarse a los constantes cambios que se producen en el mundo de la tecnología y de la información (ibidem, p. 110).

Partiendo del estudio descriptivo y contextual de los planes de estudio de dos universidades de referencia en sus respectivos países - la Fundación Escuela de Sociología y Política de San Pablo y Universidad Carlos III de Madrid-, el artículo de Funaro y Castro (2003) incide en otro aspecto complementario en los esfuerzos por adecuar la formación a los requisitos del mercado, que deben acometerse en cuatro direcciones: a) el tratamiento adecuado en el currículo de las competencias requeridas por el mercado; b) la coordinación de teoría y práctica en las asignaturas regladas; c) la valoración de la investigación como actitud profesional concretada, por ejemplo, en los proyectos de fin de carrera; y d) la presencia de un adecuado panel de optativas que faciliten una introducción suficiente a los distintos nichos de empleo. Funaro y Castro, apoyándose para España en los trabajos de Moreiro y Caridad muestran un panorama optimista de la inserción laboral y de la ampliación de los campos de acción de los titulados en Biblioteconomía y Documentación, en la dirección de la visión optimista de Cunha que traíamos a colación a principio de este apartado.

\section{La biblioteca como espacio de investigación y de convivencia}

Así pues, los cambios del último decenio - señaladamente la aceleración de la globalización y del desarrollo e impacto de las nuevas tecnologías - han sido un reto de gandes dimensiones, pero sobre todo una gran oportunidad para los profesionales de la información y la documentación - y de su enseñanza - en la medida en que han sido capaces de desarrollar las nuevas competencias que les requería el mercado de trabajo. Lejos de ser "barridos" por la revolución de las tecnologías de la información, los profesionales de la información y la documentación han encontrado un ambiente propicio para ampliar sus actividades. Incluso se podría afirmar que se muestran incapaces de dar cuenta de toda la demanda social, por lo que numerosos profesionales de otros campos están ocupando trabajos de gestores de información y documentación en sus respectivas especialidades.

Scire. 9 : 1 (en.-jun. 2003) 9-19. 
Lo mismo puede ocurrir con las instituciones más antiguas del campo, y cuya desaparición se llegó incluso a pronosticar a principios de los años noventa. No es solo que la biblioteca haya prestado su nombre a las colecciones de objetos digitales - las bibliotecas digitales - , lo cual por sí solo es muestra de la potencia conceptual que el nombre encierra, tanto más cuanto que el "bautizo" no ha sido iniciativa de bibliotecarios, sino de informáticos y comunidades de usuarios-autores. Es que la biblioteca física se está "redescubriendo" como el necesario correlato físico de su melliza "digital". La biblioteca aparece en este contexto como un "ambiente propicio" (Vieira, 2003) para la búsqueda de información; lo cual plantea un problema interesante al radicalismo de las discusiones actuales sobre el futuro de la biblioteca en el contexto de la digitalización. La biblioteca digital es una herramienta excelente, pero no un lugar; sin embargo, el ser humano vive y se desarrolla en espacios en los que se mueve, y la biblioteca está ligada a esos espacios y es en sí misma un espacio. Así, por ejemplo, es posible preguntarse: ¿Cuál es el lugar de la biblioteca digital en el proceso de formar ciudadanos? ¿El pupitre de estudio? Precisamente cuanto más se incide en el trabajo en equipo $-\mathrm{y}$ estamos viendo a nuestros estudiantes buscar la compañía de otros para afrontar su trabajo formativo a costa del hábito de estudiar en casa- más necesaria se vuelve la biblioteca como espacio de investigación, formación y de convivencia.

En el mismo sentido, no estaría mal que de vez en cuando se cambiara el término usuario o profesional de la información por el de "gente" para tomar distancia por un momento de los reduccionismos útiles que plagan nuestro discurso y nuestro pensamiento. Los protagonistas de los sistemas de información y documentación no son solo "entes inteligentes" que "usan" información y que la "sirven", sino personas que se encuentra en un espacio social para honrar con su trabajo o su afición el fruto de la labor y de las pasiones de otras personas, recogido en documentos, que son el depósito, en suma, de algunos de los valores más elevados de nuestras culturas. La biblioteca no es solo una arquitectura "clienteservidor", una estructura de servicio o una organización profesional, -que lo es-, sino también un espacio de convivencia, un lugar para el descubrimiento, una encrucijada privilegiada de los caminos del saber y del sentir. Los reduccionismos - gerencial, tecnológico, social, ético... - pueden servir para guiar la especialización y focalizar prioridades, pero a la larga solo una visión sistémica y humanística va a permitir mantener la nave de la práctica profesional, la enseñanza y la investigación de nuestro área en la estela de las disciplinas clásicas.

\section{Referencias}

Agencia Nacional de Evaluación Acreditación (2004). Título de grado en información y documentación [Recurso electrónico]. Madrid: Agencia Nacional de Evaluación

Scire. 9 : 1 (en.-jun. 2003) 9-19. 
Acreditación, 2004. URL: <http://www.aneca.es/modal_eval/docs/conver_ biblio.pdf>. Consulta: 20-08-2004.

Barcala de Moyano, Graciela G. (2003). "Memoria Abierta": patrimonio documental de organismos de derechos humanos en la Argentina. // Scire. 9:1 (en.-jun., 2003) 139-150.

Cintia Braga Ferreira; Malerbo, Maria Bernadete; Silva, Márcia Regina (2003). Errores en las referencias bibliográficas de la producción académica: un estudio de caso. // Scire. 9:1 (en.-jun., 2003) 133-138.

Cunha, Miriam Vieira da (2003). El sistema de las profesiones y el profesional de la información. // Scire. 9:1 (en.-jun., 2003) 85-93.

Fuentes-Romero, Juan José (2003). La planificación estratégica en las bibliotecas nacionales: su aplicación a la gestión del personal y a la gestión económica. // Scire. 9:1 (en.-jun., 2003) 57-74.

Funaro, Vânia Martins Bueno de Oliveira; Filho, Claudio Marcondes de Castro (2003). Comparación entre dos escuelas de Biblioteconomía de Brasil y España: aspectos curriculares. // Scire. 9:1 (en.-jun., 2003) 113-124.

García Marco, Francisco Javier (1998). Las bibliotecas y sus recursos humanos ante las nuevas tecnologías. // Asociación Andaluza de Bibliotecarios. Actas de las X Jornadas Bibliotecarias de Andalucía. Málaga: Asociación Andaluza de Bibliotecarios, 1998. 9-51.

García Marco, Francisco Javier (2003). La gestión del conocimiento: aplicación a la promoción de la salud. // Scire. 9:1 (en.-jun., 2003) 151-170.

Gilchrist, Alan (2003). Taxonomies and information architecture. // Scire. 9:1 (en.-jun., 2003) 37-46.

Guidelines on Library Legislation and Policy in Europe: Strasbourg, 23-25 November 1998 / Council of Europe; EBLIDA. URL:< http://www.coe.int/T/E/Cultural_Co-operation/ Culture/Resources/Reference_texts/Guidelines/ecubook_R3.asp\#P135_27323 >.

Leavitt H.J. (1965). Applied organisational change in industry: Structural, technological, and humanistic approaches. // March J. (ed). Handbook of Organisations. Chicago: Rand McNally \& Co., 1965. 1144-1170.

López Alonso, Miguel-Ángel (2003). Compilación de un macrotesauro conceptual para los centros españoles de información juvenil. // Scire. 9:1 (en.-jun., 2003) 47-56.

Oliveira, Ely Francina Tannuri de (2003). La formación del profesional bibliotecario en el ámbito del Mercosur: realidad y tendencias en el inicio del siglo XXI. // Scire. 9: 1 (en.-jun., 2003) 95-112.

Rodríguez Muñoz, José Vicente (2003). Información, Tecnología y complejidad. v. 2. 0. . // Scire. 9:1 (en.-jun., 2003) 23-36.

Vergueiro, Waldomiro; Carvalho, Telma de (2003). Programas de Calidad en Bibliotecas Brasileñas: Panorama y Perspectivas. // Scire. 9:1 (en.-jun., 2003) 75-83.

Vieira, Kátia Corina (2003). Investigación y construcción del saber en la Nueva Escuela: el papel de la biblioteca escolar. // Scire. 9:1 (en.-jun., 2003) 125-132. 